\title{
Study of Linear and Decision Feedback Equalizers on Fading Channels
}

\author{
Zeeshan A. Abbasi \\ Assistant Professor \\ University Polytechnic \\ Jamia Millia Islamia, New Delhi
}

\author{
Zainul Abdin Jaffery \\ Professor \\ Dept. of Electrical Engineering \\ Jamia Millia Islamia, New Delhi
}

\begin{abstract}
For evaluating the performance of wireless digital communication system the most important part is the simulation of wireless channel, as whole performance of the system is depends upon it. The Band limited communication channel, due to its dispersive time response, may restrict the high data rate, due to Inter symbol Interference (ISI). If the ISI is not taken care off and compensated may cause high Bit Error Rate (BER). Equalizers are used for this purpose. In this paper the performance of Linear and Decision Feedback Equalizers (DFE) are evaluated on three wireless channels i.e. two ray path, Rayleigh and Rician. A model of communication system having Quadrature Amplitude Modulation (QAM) with fading channels is implemented using MATLAB. The performance of Linear and DFE is evaluated on the basis of Bit Error Rate (BER) with varying Signal to Noise Ratio (SNR). The simulation results showed the performance of two equalizers on different channels. The Rayleigh channel model is the most suitable model for urban areas as it is the model which simulates a channel with no Line of Sight (LOS) path and is the most difficult environment.
\end{abstract}

\section{General Terms}

ISI (Inter-symbol Interference), BER (Bit Error Rate), SNR (Signal to Noise Ratio)

\section{Keywords}

Equalization, Fading Channel, DFE

\section{INTRODUCTION}

Wireless communication has become a most important area of technology development as it is a prominent part of everyday life. Due to the increasing demand of data communication through mobile or wi-fi, wireless channels plays an important role as they induced distortion results in Inter Symbol Interference (ISI), which if left uncompensated can cause higher error rates. The best compensation for ISI problem is the equalizer. To design a good equalizer we must have to model the channel accurately. The three important channel models to describe a time-variant nature of a channel, frequently used, are two rays, Rayleigh and Rician [1].

Fading or loss of signals is a very important phenomenon that revealed to the wireless communication field. The better a model can describe a fading environment the better it can be compensated at the receiver with the help of equalizer, as an equalizer is an inverse filter which models a channel [2]. Our purpose is to receive a signal at the receiver error free or close to being error free. It would result in better clarity of voice in case of audio transmission or higher accuracy of data in case of data transmission. Therefore in a wireless communication system the selection of fading model and equalizer plays a very important role [2].

\section{WIRELESS CHANNEL MODELING}

A wireless channel may be modelled as a linear filter with a time varying impulse response, where the time variation is due to receiver motion in space. Due to the different multipath waves which have propagation delays which vary over different spatial locations of the receiver, the impulse response of the LTI channel should be a function of the position of the receiver.

$y(d, t)=x(t) * h(d, t)=\int_{-\alpha}^{\alpha} x(\tau) h(d,(t-\tau)) d \tau$

$h(d, t)$ is the channel impulse response.

$x(t)$ is the transmitted signal.

$y(d, t)$ is the received signal at position " $\mathrm{d}$ ".

\subsection{Fading and Multipath}

In wireless communication system fading is due to the multipath i.e the received signal reaching the receiver antenna by two or more path. Due to the result of fading the received signal includes multiple versions of the transmitted signal which are attenuated and delayed in time [4]. A multipath channel induces Inter Symbol Interference (ISI). According to the effect of multipath the fading can be classified as:

(a) Large scale Fading in which received signal power varies due to attenuation in the signal.

(b) Small scale Fading results due to rapid fluctuations of phase and amplitude of the signal.

\subsection{Types of Small Scale Fading}

\subsubsection{Two Ray Model}

In two ray model the two paths are Line of sight (LOS) and ground reflected wave. This is the simplest model used for wireless fading model. Figure 1 shows a two ray model. 


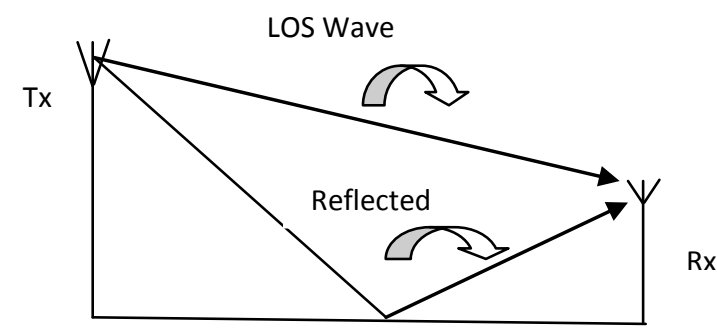

Fig. 1: Two Ray Path Channel

\subsubsection{Rayleigh Fading Model}

It is the model which describes the effect of heavily built up urban environment onwireless signal. It is most applicable when there is no line of sight (LOS) path or a direct path between transmitter and receiver.

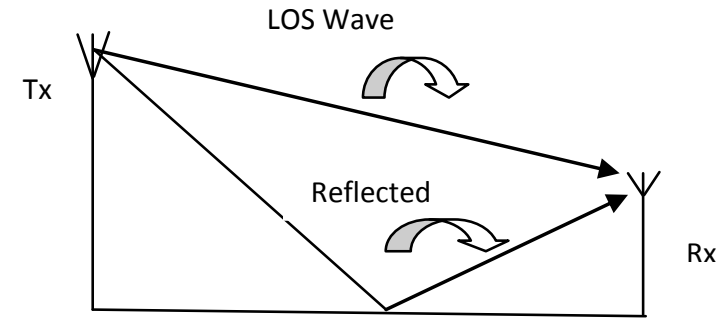

Fig. 1: Two Ray Path Channel

\subsubsection{Rician Fading Model}

It is similar to the Rayleigh fading model with only the difference that in Rician fading there is a strong dominant component known as LOS component.

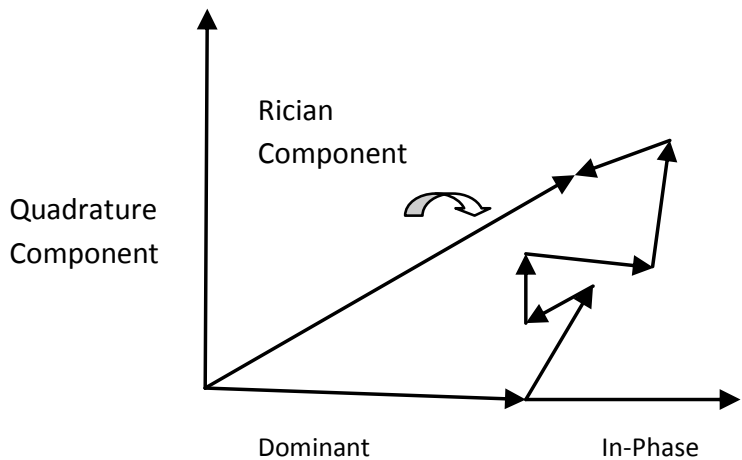

Fig 3: Rician Signal Model

\subsubsection{AWGN Model}

This is the simplest radio environment which added the background noise to the transmitted signal thus received signal $r(t)$ can be written as $r(t)=x(t)+n(t)$ where $x(t)$ is the transmitted signal and $\mathrm{n}(\mathrm{t})$ is the background noise. This channel added the while Gaussian noise to the signal that passes through it and used as a standard channel model.

\section{STRUCTURE OF THE MODEL}

Figure 4 shows the model of the communication system with linear equalizer. It consist of random integer generator, QAM Modulator, Fading Channel (Two Ray/Rayleigh/Rician), equalizer (Linear/DFE), Gain control and QAM demodulator [3]. For simulation purpose one million bits are generated with equal probability of +1 and -1 . These bits are modulated using 16 QAM. These modulated bits are passed on to the fading channel. AWGN noise is also added. The equalizer is implemented in the receiver. The received signal is equalized and then de-modulated in case of linear equalizer (Figure 4). The received bits after hard decision are comparing with the generated bits for the purpose of BER calculation. Figure 5 shows the model with DFE [5]. All the blocks are same as used in linear equalizer except gain control and training sequence transmitter and receiver. The gain control block controls the overall gain of the channel. For simulation of DFE equalizer a training sequence is generated and transmitted prior to message signal so that the equalizer automatically adjusts its parameters in accordance with the estimation error [6]

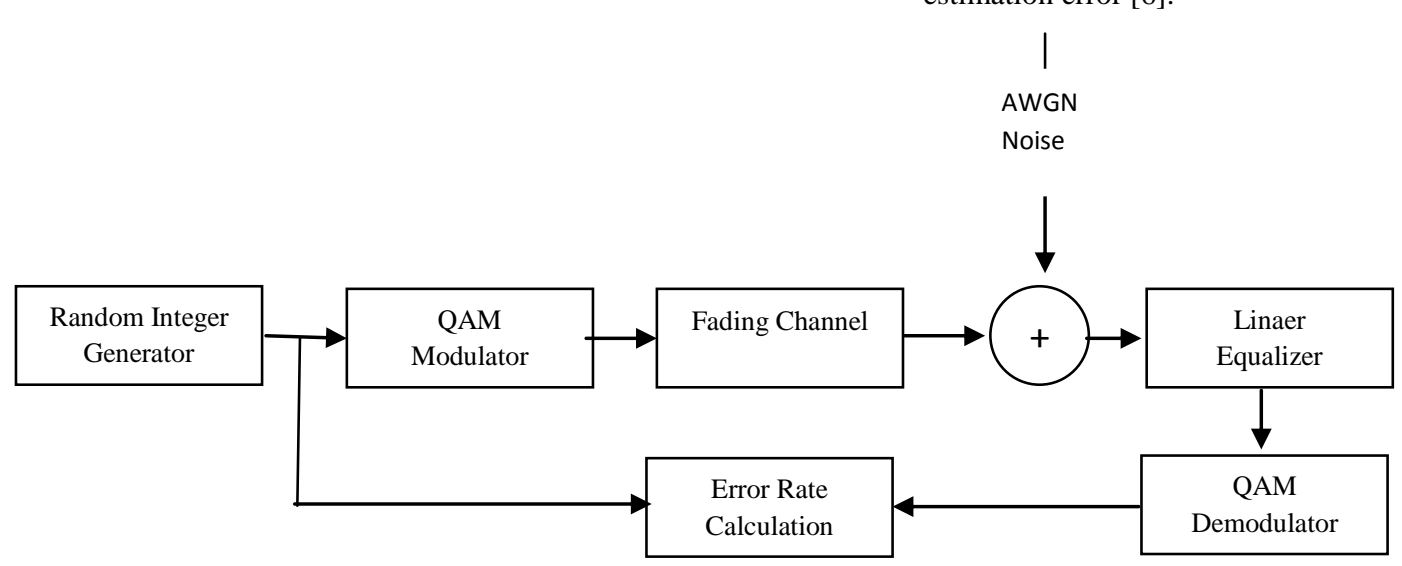

Fig. 4: Structure of Linear Equalizer 
The estimation error is calculated by comparing the received signal from the channel with the signal generated by training sequence $\mathrm{Rx}$ which is the replica of transmitted training sequence. The BER can be calculated by comparing the received bits after equalizer with the transmitted bits and dividing the total numbers of unequal pairs of data elements by the total number of input data.

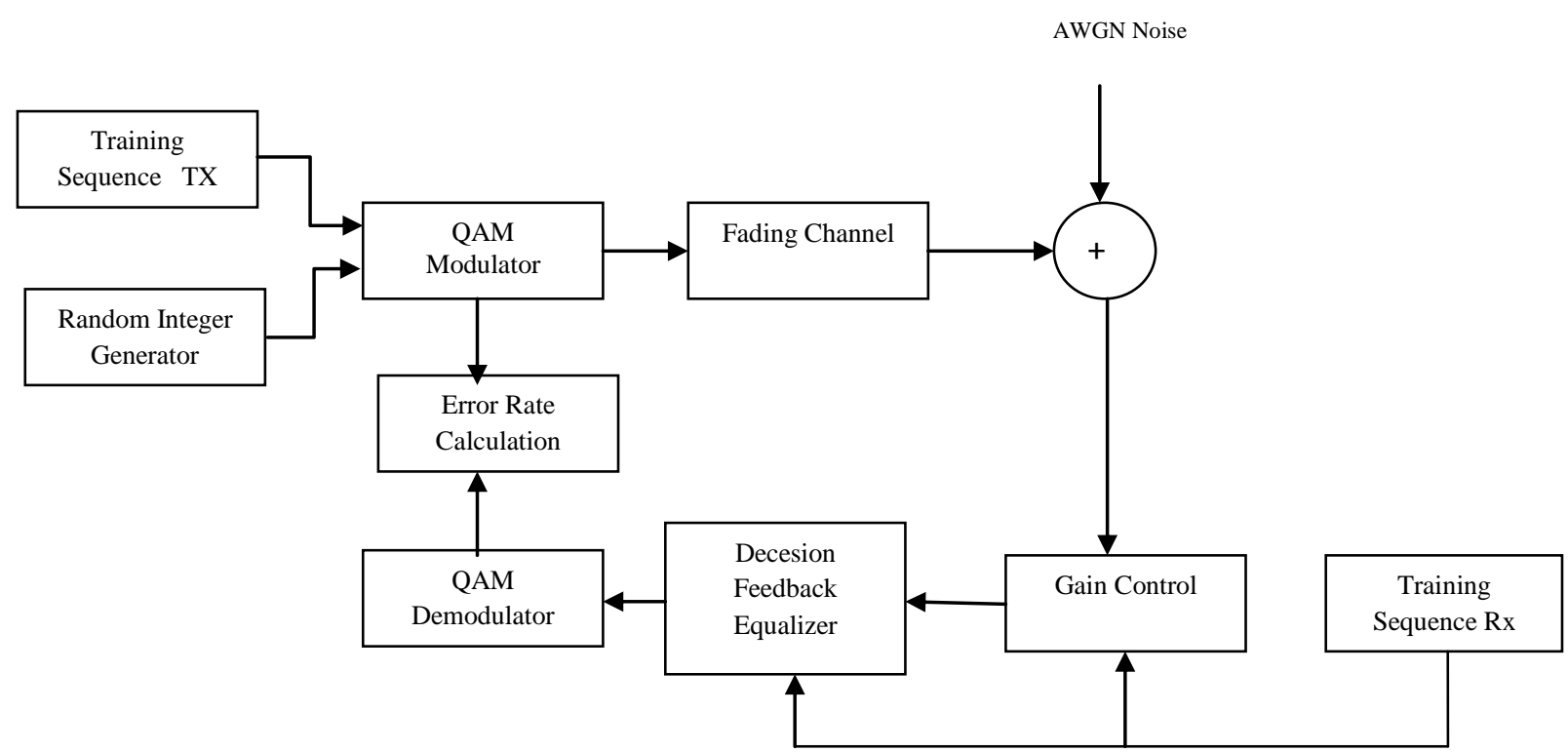

Fig. 5: Structure of Decision Feedback Equalizer

\section{RESULTS ANALYSIS}

In this paper the different fading environment is implemented as we encountered in our daily life wireless communication with the help of MATLAB functions. The ISI introduced by these channels are compensated by Equalizers and BER is computed. Due to its easy scripting and excellent data visualization capability the MATLAB is an ideal tool for simulating various communication systems and channels. Bit Error Rate is the figure of merit of all communication system. The binary data is generated and modulated by 16 QAM modulations. The figure 6 shows the constellation diagram of the modulated data. Figure 7 shows the constellation diagram of the data received before and after equalization. It is clear from the figure 7 that data can be received easily in case of two rays but not in case of Rician and Rayleigh channel. It is also clear from the figure 8 that the BER is very close to one in case of Rician channel with linear equalizer and almost one in case of Rayleigh channel means linear equalizer completely failed on Rayleigh channels as $100 \%$ bits are in error. With Decision feedback equalizer the BER is sharply reduced with Rician channel but to get the same BER on Rayleigh channel almost double signal to noise ratio is required.

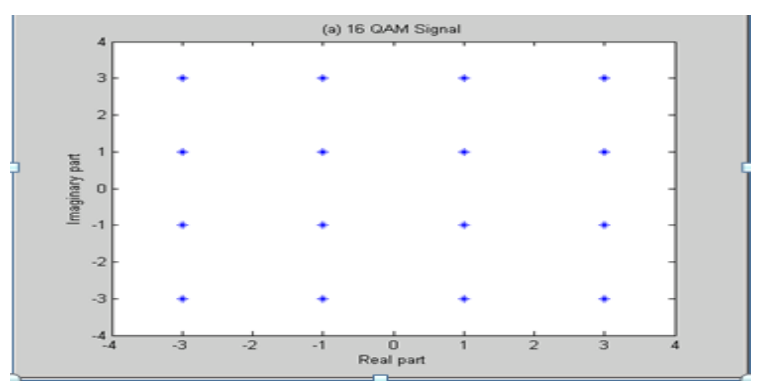

Fig. 6: Constellation Diagram for 16 QAM Modulation

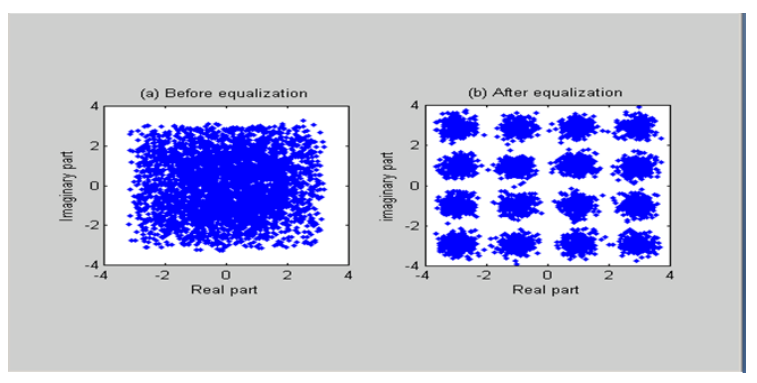

(a)

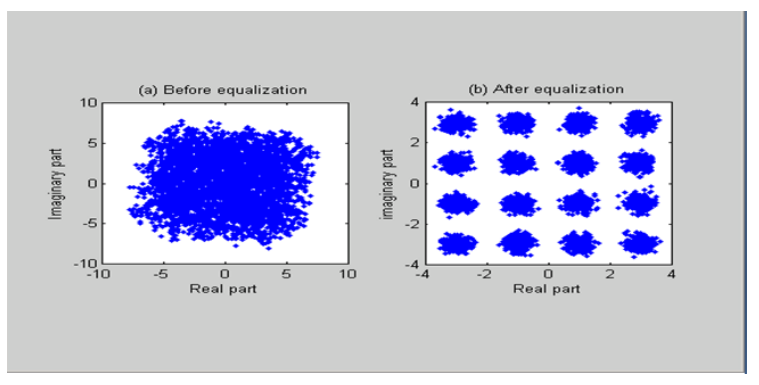

(b)

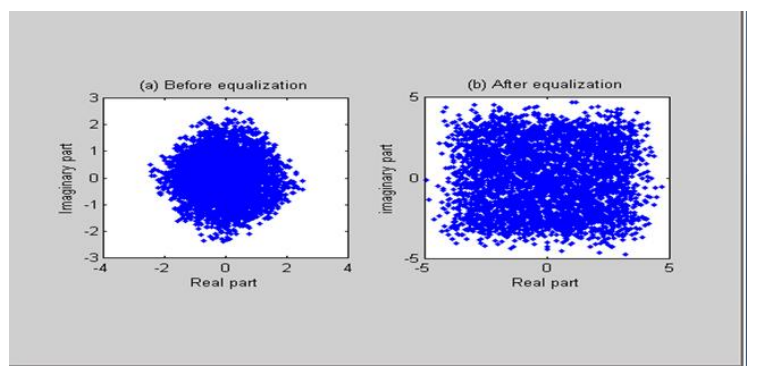

(c)

Fig. 7: Data before and after equalization for (a) Two 
path channel (b) Rician Channel (c) Rayleigh Channel

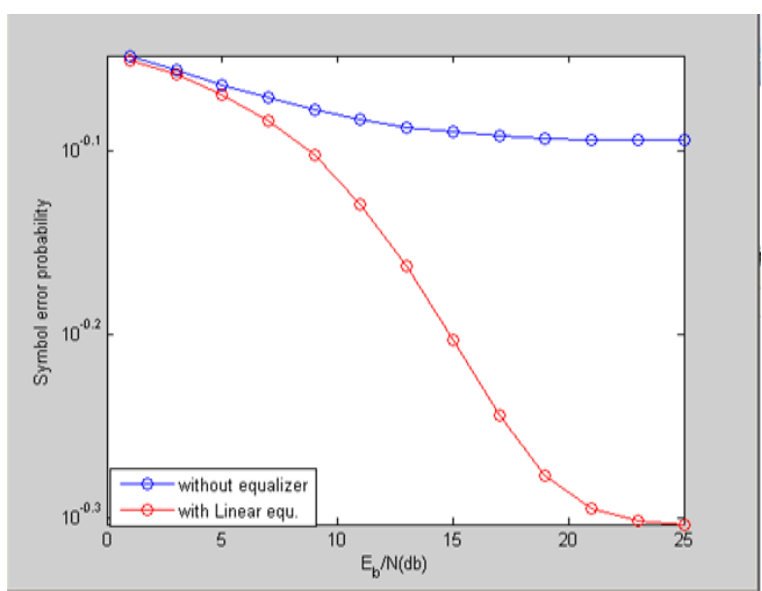

(a)

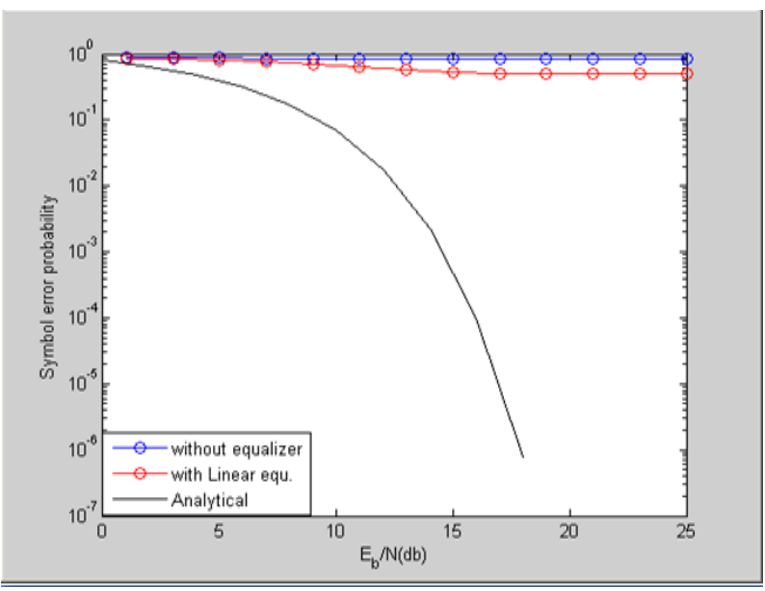

(b)

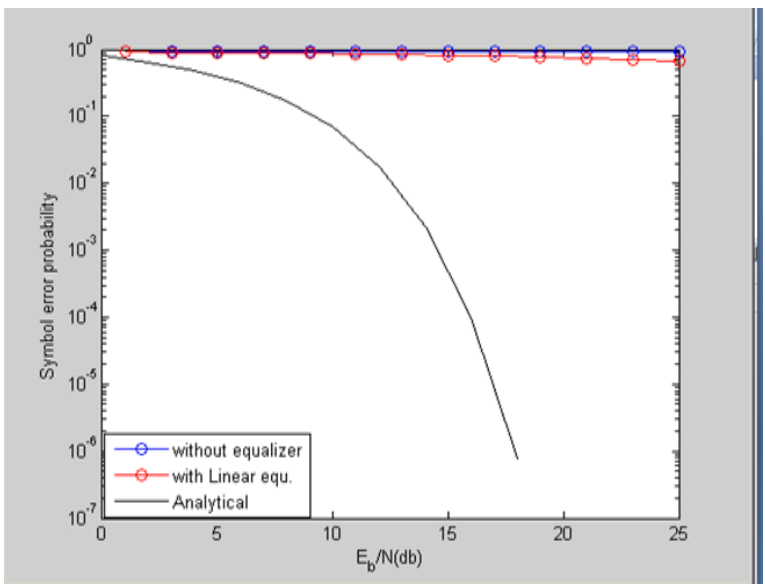

(c)

Fig. 8: Response of Linear equalization for (a) Two path channel (b) Rician Channel (c) Rayleigh Channel

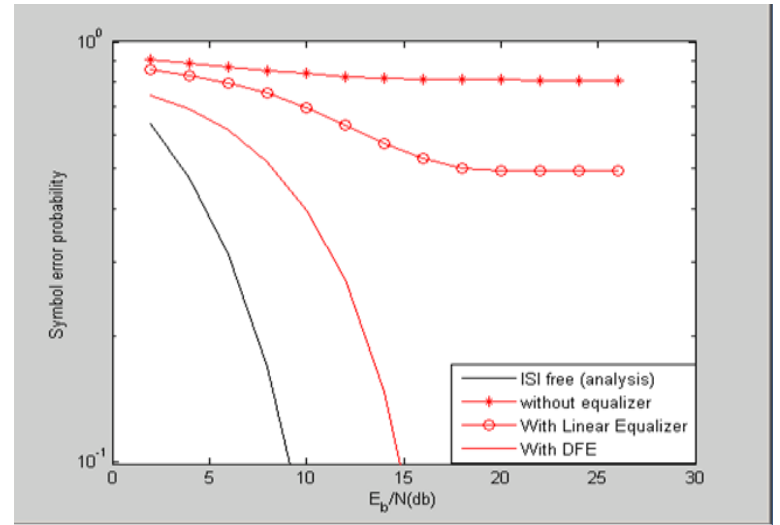

(a)

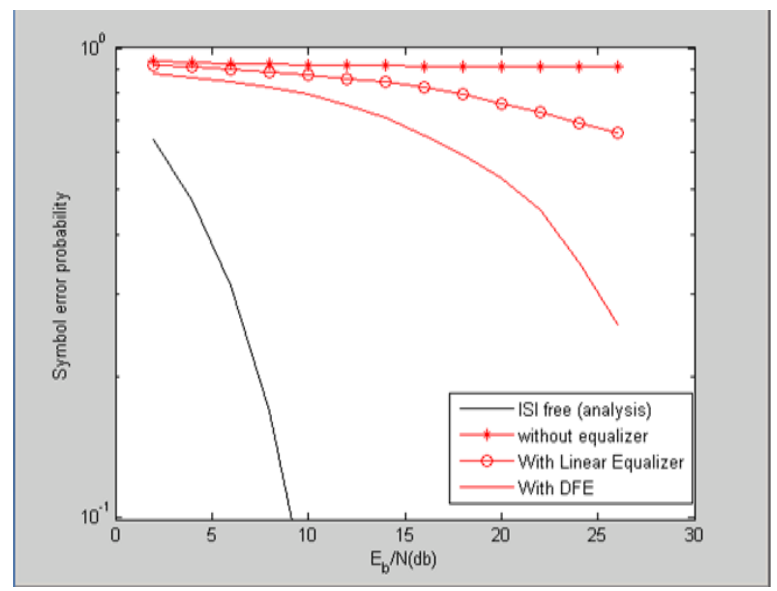

(b)

Fig. 9: Response of DFE equalization for (a) Rician Channel (b) Rayleigh Channel

\section{CONCLUSION}

In this paper performance of linear and DFE is evaluated on different fading channels. The analysis showed that Linear equalizers perform better on two ray channel but failed on Rician and Rayleigh fading channels. The DFE performance very well on Rician as it has a LOS path which gives more SNR and less BER but still not satisfactory at Rayleigh as Rayleigh fading channel is much more difficult environment than others.. In case of Rayleigh 15 DB extra (15 times higher) power is needed to get the good results. The paper proposed en elementary idea of equalizer on fading channel the same idea can be implemented using other techniques such as Neural Networks and Fuzzy System.

\section{REFERENCES}

[1] M. K. Simon and M. S. Alounini, "Digital Communication Over Fading Channels: A Unified Apprpach to Performance Analysis", Wiley, New York 2000.

[2] Upena Dalal, "Wireless Communication and Networks", Oxford University Press, 2015.

[3] Mahmood Farah Mosleh and Aseel Hameed AL Nakkash, "Combination of LMS and RLS Adaptive Equalizer for Selective Fading Channel", European Journal of Scientific Research, Vol. 43 No 1,2010 pp $127-137$ 
[4] A. Sudhir Babu and Dr K. V. Sambasive Rao, "Evalution of BER for AWGN, Rayleigh and Rician Fading Channels Under Various Modulation Schemes", International Journal of Computer Applications, Volume 26-No.9, July 2011.

[5] Sharma O.P, Janyani V. And Sancheti S., "Recursive
Least Square Adaptive Filter a better ISI Compensator", World Academy of Science and Technology 52, 2009, pp 1031-1036.

[6] Garima Malik and Amandeep Singh Sappal, "Adaptive Equalization Algorithm: An Overview", International Journal of Advance Computer Science and applications, Vol. 2, No. 3 March 2011 pp 62-67. 